\title{
Recent Advances in the Chemistry of the Vitamins.
}

$A^{\mathrm{T}}$ the meeting of the Royal Society held on June 18, the President, Sir Frederick Gowland Hopkins, opened a discussion on the chemistry of the vitamins. He said that although many discoveries had been communicated in the past to the Society, there had been very few papers dealing with the vitamins. He felt great satisfaction that this discussion should occur during the first year of his presidency, especially as the subject was still growing in interest. The Society were to be congratulated on the presence of a number of foreign workers, so that the discussion would have an international character. As the subject was so vast, he proposed to limit it to the chemistry of the vitamins, and suggested that vitamins $D, A$, and the $B$ complex be taken in that order.

\section{Vitamin D.}

Prof. A. Windaus said that up to the present, investigations had had to be carried out on impure substances and chiefly by means of physical experiments. No stable equilibrium was formed between ergosterol and the products of irradiation. By further irradiation two crystalline substances could be obtained, neither of which could be converted into the other; it appeared therefore that two series of products were formed on irradiation. Reerink and van Wijk had found that no matter whether 10 or 50 per cent of the ergosterol was changed by long wave irradiation, the absorption spectrum of the product was always the same. $\mathrm{He}$ suggested that this was due to several substances being formed in constant proportion, and that the absorption spectrum was that of the mixture. None of the produets of irradiation are precipitated by digitonin. Failure of precipitation, however, does not imply that a change has taken place in the hydroxyl group. On treatment with phenyl isocyanate the antirachitic activity of the irradiated ergosterol is destroyed. Treatment with warm caustic potash results in the reconversion of the phenyl urethane to vitamin $\mathrm{D}$.

Prof. Windaus had found that the vitamin had the same molecular weight and formula as ergosterol and also contained three double bonds. The dihydro derivative obtained from irradiated ergosterol by treatment with sodium in alcoholic solution was inactive, but it is not certain that it is a derivative of vitamin $\mathrm{D}$ itself. The vitamin is more sensitive to a temperature of $180^{\circ}$ than ergosterol after heating, the absorption spectrum shows a band at $2820-2920 \mathrm{~A}$.

Crude vitamin $\mathrm{D}$ is stable in oil, although the absorption spectrum and specific rotation rapidly change. The toxicity of the crude product varies with the potency, but it is still possible that the two properties may be due to different substances. The problem has not yet been solved. He had noticed that in vacuum tubes, in which crude vitamin $D$ had been sealed, crystals appeared, but he had not been able to obtain them in a pure state by recrystallisation from cold acetone or by fractional precipitation. He had found, however, that when irradiated ergosterol was treated with maleic or citraconic anhydride in ethereal solution at room temperature for one to three days, a reaction occurred between certain of the substances present and the anhydride. When these inactive products were removed by solution in dilute caustic potash, crystals could be obtained from the ethereal layer on evaporation of the solvent. The yield was 50 per cent of the crude product, or
60-70 per cent of the material which failed to react with maleic anhydride.

Vitamin D crystallises in long needles of melting point $122^{\circ} ;[a]_{\mathrm{D}}{ }^{18}=+136^{\circ}$ in acetone, and $[\mathrm{a}]_{\mathrm{Hg}}{ }^{18}$ $=+168^{\circ}$ in acetone.

The crystals show a band in the absorption spectrum at $2650-2700 \mathrm{~A}$. Their potency was found to be $2-2 \frac{1}{2}$ times that of the M.R.C. standard.

He considered that the product obtained by Reerink and van Wijk was different from his, since it had a lower specific rotation and an absorption spectrum of a different shape, but that Bourdillon's crystals were probably the same. He agreed with the suggestion of the latter that there might be several compounds showing vitamin D activity, but considered that his crystals were responsible at any rate for the chief part of it. Vitamin D is an isomer of ergosterol in which there has been a structural rearrangement with an increase in the spatial size of the molecule.

Prof. B. C. P. Jansen said that Reerink and van Wijk had now obtained crystals of a melting point $140^{\circ}$. Their former product with a lower melting point had contained ether of crystallisation.

Dr. R. B. Bourdillon said that the discovery of the method of obtaining crystals of vitamin $D$ by means of maleic anhydride was of outstanding importance and likely to lead to a solution of the problem of its constitution. $\mathrm{He}$ and his co-workers had obtained very similar crystals by distillation of irradiated ergosterol in a high vacuum. Their melting point was $123-125^{\circ}$. The specific rotation to the mercury line in alcoholic solution was +250 to $+260^{\circ}$. The maximum absorption occurred at $2700 \mathrm{~A}$. and was greater than that of ergosterol. The potency was 18-22,000 M.R.C. U./mgm., that is approximately the same or slightly less than that shown by Prof. Windaus' crystals. They had been able to prepare an oxalate and acetate and reconvert these back to the original crystals. They had found the rotation and potency to remain constant for some weeks in dry air or in vacuo. Neither by distillation at a temperature of $160^{\circ}$ nor by further irradiation with loss of two-thirds of the potency had they been able to separate their crystals into two separate compounds. Moreover, on irradiation the loss of antirachitic activity and the changes in the absorption coefficient and specific rotation were absolutely parallel. They therefore considered that their crystals were a definite chemical compound and had ventured to call it 'Calciferol'. However, the unity of the compound was not absolutely certain, owing to variations in the specific rotation of different preparations. They had recently obtained some crystals with a rotation of $+290^{\circ}$. He suggested that there were at least two vitamins, isomorphic and with the same absorption spectra, the lævo form being very unstable, while the dextro form was stable. This suggestion would explain the discrepancies in his own work and that of other observers.

Dr. O. Rosenheim said he considered the work now reported was the most important since the original researches on vitamin $\mathrm{D}$. He thought that Windaus' and Bourdillon's crystals were identical, although it was possible that there might be an impurity present as in Reerink and van. Wijk's preparation. Spectroscopic methods had not led to much advance, but success had come from biological and organic chemical research, and especially from Bourdillon's method of fractional condensation. He pointed out that if ergosterol was irradiated until 20 per cent had been changed and the 80 per cent of inactive material 
removed, the activity of the residue was only the same as that of the original irradiated material. On distillation of the residue, 20 per cent was obtained as crystals, but the activity was only twice that of the crude product.

\section{VITAMIN A.}

Prof. H. von Euler said that it was early noticed that fat soluble growth-promoting material was frequently associated with a red or yellow coloration but that the converse was not true. Working with Karrer, he had found that of all red or yellow substances examined, only carotene had vitamin A activity. Carotene was usually supposed to be optically inactive, but he, as well as Rosenheim and Kuhn working independently, had found that it could be fractionated into two forms, one melting at $170^{\circ}$ and optically active, and the other melting at $183^{\circ}$ and optically inactive. Both forms had growth-promoting power. The latter gave an earlier growth response, but after three weeks the differences between the two disappeared. A dose of $0.003 \mathrm{mg}$. daily would produce a daily increase in weight of one gram in the rat. Complete hydrogenation of carotene inactivated it, but reduction with aluminium amalgam at first increased the activity. Hydrocarotene containing eight double bonds is much more active than carotene, the daily dose required being only $0.0005 \mathrm{mg}$. It also gives a higher blue value with the Carr and Price colour test. The absorption spectrum is very similar to that attributed to vitamin $\mathrm{A}$ in cod liver oil.

Moore considers that carotene is converted to vitamin A in the body of the rat. Prof. von Euler had tried to effect this conversion in vitro, but only with the serum of the hen had he found that carotene could be converted into a substance very similar to vitamin $A$ although not spectroscopically identical. He considered it probable that the transformation occurs in the blood, and that vitamin A may act as a catalyser in oxidation. $\mathrm{He}$ had also examined the anti-infective action of carotene and had found that it did not affect hæmolysis in vitro nor react with amboceptor, but that when it was given in excess to rabbits, the amboceptor in their blood was increased.

Dr. Rosenheim said that carotene is a mixture which has not yet been completely separated, and that it is dangerous to attribute activity to any particular isomer. The optical activities obtained by different observers vary considerably. It is possible that the transformation of carotene into vitamin $A$ in the body may be only the accumulation of vitamin A present as a contaminant in the carotene. The band in the absorption spectrum of cod liver oil at $3280 \mathrm{~A}$. may not be due to vitamin A but to a substance accompanying it.

Dr. R. A. Morton said that if the band at $3280 \mathrm{~A}$. is to be attributed to vitamin $A$, then the latter cannot be present in carotene as an impurity. Dihydrocarotene is not vitamin $\mathrm{A}$, since the band in its absorption spectrum is at $3170 \mathrm{~A}$., the blue colour given with antimony trichloride is not the same as that given by the vitamin, and the ratio of blue colour to intensity of absorption is different.

\section{Vitamin B.}

Prof. B.C. P.Jansen said that when $100 \mathrm{kgm}$. of rice polishings were extracted with dilute acid alcohol, $30 \mathrm{kgm}$. went into solution. By treatment with acid clay it was possible to adsorb nearly all the vitamin $B_{1}$, but only 100 grams of contaminating solid material. The vitamin $\mathrm{B}_{1}$ present accounted for only 1 per cent of the adsorbed material. By fractional precipitation with silver nitrate and baryta it was possible to remove impurities at a strongly acid reaction and to precipitate two-thirds of the vitamin at $p \mathrm{H}$ 4-7: above this $p \mathbf{H}$ impurities were precipitated as well.

Further purification could be effected by precipitation with phosphotungstic acid, and decomposition of the precipitate with baryta, and precipitation with platinum chloride from alcoholic solution. When the platinum was removed with powdered silver, 1.4 gram was obtained, of which 0.4 gram was pure vitamin. By numerous fractionations with acetone from solution in absolute alcohol, $30 \mathrm{mgm}$. of pure vitamin were obtained. The formula by analysis was $\mathrm{C}_{6} \mathrm{H}_{10} \mathrm{ON}_{2} \mathrm{HCl}$. The process he had devised was therefore very wasteful. It could be improved by the use of Peters' method of fractional precipitation with phosphotungstic acid. Prof. Jansen had found that silicotungstic acid was as suitable. When the original 100 grams obtained by adsorption on acid clay were treated with this reagent, two-thirds of the vitamin were precipitated, but only 20 per cent of the total solid. A further improvement had been effected by Seidell's benzoylation process, which removes impurities. By this method von Ween had obtained $140 \mathrm{mgm}$. of pure vitamin $\mathrm{B}_{1}$ from $75 \mathrm{kgm}$. rice polishings.

Prof. A. Seidell said that Prof. Jansen had had success with a method that had failed in his hands. He had felt that precipitating agents ought to be avoided, and so was led to the method of benzoylation in chloroform solution, which removes a considerable amount of nitrogenous material. He had not succeeded in obtaining crystals, although his purest preparation was nearly as active on rats, but when tested on pigeons by Peters' method the activity was found to be only $1 / 5$ to $1 / 10$ that of the crystals.

Prof. R. A. Peters said that he had hoped to be able to use the animal as a test object only and not concern himself with the physiological side of the problem, but he had found that it was only possible to reach a final conclusion on the chemistry when the physiology was also taken into account. He had been able to confirm Prof. Jansen's process up to the platinum chloride stage. Working with yeast he had obtained purification by removal of inactive material with lead acetate and baryta followed by adsorption of the vitamin by charcoal. By fractional precipitation with phosphotungstic acid at $p \mathrm{H} 5-7$, vitamin $B_{1}$ was obtained. Its activity was $0.012 \mathrm{mgm}$. per dose, while that of Prof. Jansen's crystals in his hands was $0.008 \mathrm{mgm}$. Both preparations gave the Pauly reaction with the same intensity. Miss Reader had shown that 25-50 per cent of Prof Jansen's crystals had vitamin $B_{4}$ activity. She had now been able to isolate vitamin $B_{1}$ from $B_{4}$ by fractional adsorption on to charcoal in the earlier stages of the purification, but the separation of $B_{4}$ from $B_{1}$ was less complete.

Dr. B. C. Guha said that he had been able to separate $B_{1}$ from $B_{2}$ by electrodialysis. Vitamin $B_{1}$ appeared to be a strong base, while $B_{2}$ was a neutral substance.

The President, in summing up the discussion, said that the progress of the last few years should make them optimistic. He expected that we should soon know the constitution of vitamin D. The chemical problems involved were a priori difficult, but workers were finding that the compounds were amenable to the methods of organic chemistry. He thanked the visitors from overseas for taking part in the discussion.

$$
\text { No. } 3218 \text {, Vol. 128] }
$$

